\title{
Case report: Optimizing intraoperative detection of pulmonary embolism using contrast-enhanced echocardiography
}

\author{
[Présentation de cas : optimaliser la détection peropératoire de l'embolie pulmo-
}

naire au moyen de l'échocardiographie de contraste]

Igor Izrailtyan MD, ${ }^{*}$ Jeffrey Clark MD, * Madhav Swaminathan MD, ${ }^{*}$ Mihai V. Podgoreanu MD, * Burkhard Mackensen MD, ${ }^{*}$ R. Duane Davis MD, $\dagger$ Joseph P. Mathew MD*

\begin{abstract}
Purpose: Perioperative pulmonary embolism (PE) is associated with significant morbidity and mortality. Intraoperatively, the clinical management of patients with PE can be enhanced by the use of transesophageal echocardiography (TEE) to visualize emboli, assess pulmonary artery (PA) anatomy, and monitor the function of the right ventricle. However, the sensitivity of intraoperative TEE to detect thromboemboli is reported to be below $50 \%$. In this report, we describe the use of contrastenhanced TEE (CE-TEE) to improve the visualization of PE.
\end{abstract}

Clinical features: A 44-yr-old female with chronic thromboembolic pulmonary hypertension was scheduled for pulmonary thromboendarterectomy. The precardiopulmonary bypass TEE exam demonstrated signs of PA obstruction and right ventricle dysfunction, but the borders of the thrombus in the right PA were only minimally visualized. Perflutren lipid microspheres, composed of octafluoropropane encapsulated in an outer lipid shell, were injected as a $0.3 \mathrm{~mL}$ iv bolus, while visualizing the right PA with harmonic ultrasound imaging. The CE-TEE image clearly visualized a large mobile thrombus along with a distinct pattern consistent with pulmonary flow obstruction. The postcardiopulmonary bypass CE-TEE confirmed thrombus evacuation and absence of PA flow abnormalities.

Conclusion: Contrast-enhanced-TEE may decrease operator dependency and increase the sensitivity necessary to detect central, surgically accessible PE.

Objectif : L'embolie pulmonaire (EP) périopératoire est associée à une morbidité et à une mortalité significatives. La prise en charge peropératoire des patients qui présentent une EP peut être améliorée avec l'usage de l'échocardiographie transœsophagienne (ETO) pour visualiser l'embole, évaluer l'anatomie de l'artère pulmonaire (AP) et surveiller la fonction du ventricule droit. La sensibilité de I'ETO peropératoire à détecter la thromboembolie est cependant de moins de $50 \%$. Nous décrivons l'usage de l'ETO de contraste (ETO-C) pour améliorer la visualisation de l'EP.

Éléments cliniques: Une femme de 44 ans, souffrant d'hypertension pulmonaire thromboembolique chronique, devait subir une endartérectomie pulmonaire. L'examen ETO fait avant la circulation extracorporelle a démontré des signes d'obstruction de l'AP et de dysfonction du ventricule droit, mais les limites du thrombus dans I'AP droite n'étaient que minimalement visibles. Des microsphères lipidiques de perflutren, composées d'octafluoropropane encapsulé dans une coquille lipidique, ont été injectées en un bolus de 0,3 $\mathrm{mL}$ iv tout en observant l'AP droite avec l'échographie harmonique. L'image de I'ETO-C a clairement montré un gros thrombus mobile ainsi qu'une structure particulière caractéristique d'une obstruction du débit pulmonaire. L'examen ETO-C fait après la circulation extracorporelle a confirmé l'évacuation du thrombus et l'absence d'anomalies du débit de l'AP.

Conclusion : L'ETO de contraste peut diminuer la dépendance à l'opérateur et augmenter la sensibilité nécessaire pour détecter une EP centrale, chirurgicalement accessible.

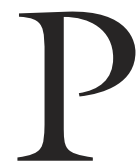
ULMONARY embolism (PE) is associated with significant morbidity and mortality. ${ }^{1}$ Surgery constitutes a significant risk for developing this life-threatening condition with some studies reporting that up to $17 \%$ of perioperative PE occur during surgery. 2,3 Another scenario encountered intraoperatively is that of a patient undergoing emergent pulmonary embolectomy, a procedure reserved for those with acute massive PE, who develop hemodynamic instability despite maximal medical therapy, and/or when thrombolysis is

From the Departments of Anesthesiology* and Surgery, $†$ Duke University Medical Center, Durham, North Carolina, USA.

Address correspondence to: Dr. Joseph P. Mathew, Department of Anesthesiology, Box 3094, Duke University Medical Center, Durham,

NC 27710, USA. Phone: 919-681-6752; Fax: 919-681-4978; E-mail: mathe014@mc.duke.edu

Accepted for publication January 6, 2006.

Revision accepted February 13, 2006.

Competing interests: None declared. 


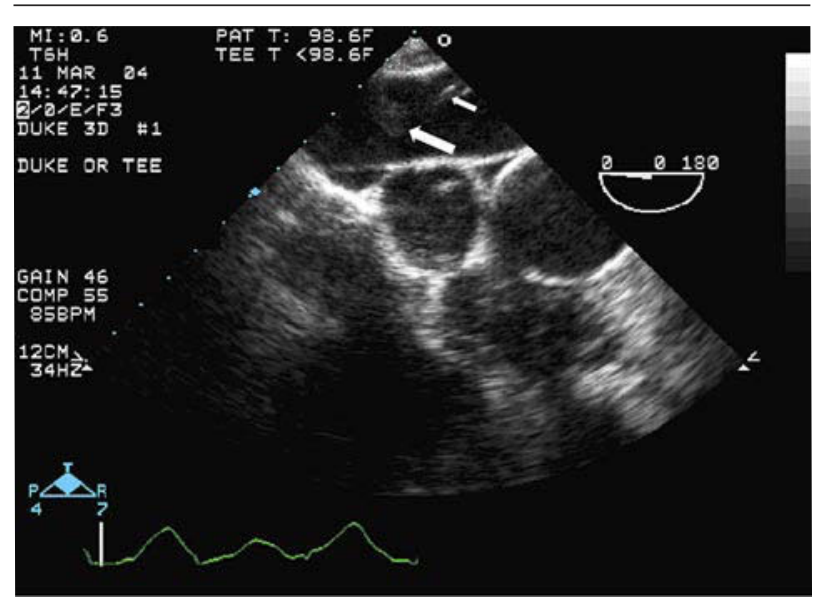

FIGURE 1 Precardiopulmonary bypass two-dimensional imaging of a thrombus (large arrow) in the right pulmonary artery. A reverberation artifact (small arrow), presenting as small linear echoes parallel to the endovascular border of the right pulmonary artery, is also seen in this image.

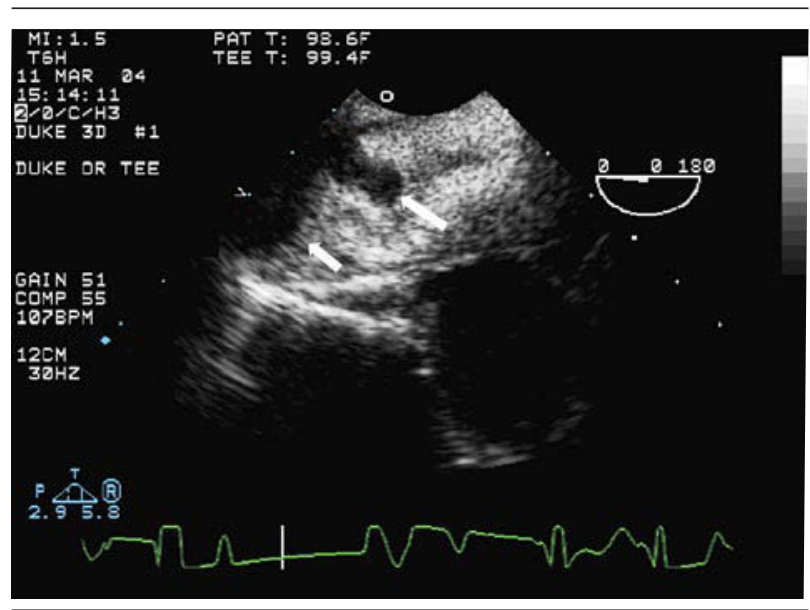

FIGURE 2 Precardiopulmonary bypass harmonic mode imaging with contrast enhancement clearly demarcating the borders (arrows) of the thrombus. A swirling flow pattern suggestive of partial obstruction was also seen. contraindicated. ${ }^{4}$ A third group of PE-patients seen in the operating room are those with chronic PE. Pulmonary thromboendarterectomy is an option for these patients who often also present with pulmonary hypertension..$^{5,6}$ Intraoperatively, the clinical management of patients with PE can be improved by the use of transesophageal echocardiography (TEE) to visualize emboli, assess pulmonary artery (PA) anatomy, and monitor the function of the right ventricle (RV). However, the sensitivity of intraoperative TEE to detect thromboemboli was recently reported to be only $46 \%{ }^{7}$ In this case report, we describe the use of contrast-enhanced TEE (CE-TEE) to image a pulmonary thrombus in a patient presenting for pulmonary thromboendarterectomy.

\section{Case presentation}

Presentation of this case was approved under the guidelines established by the Institutional Review Board at Duke University Medical Center. A 44-yrold female was admitted to the hospital complaining of right-sided chest and back pain, cough, and shortness of breath on exertion. Six weeks earlier she was hospitalized for the first time, diagnosed with chronic thromboembolic pulmonary hypertension, antiphospholipid syndrome, and protein $\mathrm{C}$ deficiency, and anticoagulated with warfarin. During the current hospitalization, she required supplemental oxygen and was noted to have sinus tachycardia with a RV strain pattern on electrocardiogram, and chest $x$-ray-changes suggestive of PA enlargement. Spiral computerized tomography (CT) demonstrated prominent clot within the right PA (RPA), extending to the right lower lobe segmental arteries, and a thin rim of clot within the left PA. There was also marked enlargement of the right heart chambers, a moderate pericardial effusion, and areas of infarction in the right lower lobe. Subsequent to transthoracic echocardiography, which revealed a hypokinetic $\mathrm{RV}$, moderate tricuspid regurgitation (TR), and PA systolic pressure of $91 \mathrm{mmHg}$, a surgical plan for pulmonary thromboendarterectomy was developed. Prior to operation, pulmonary angiography confirmed a large proximal thrombus in the RPA but predominantly small arterial occlusions on the left. During angiography, an inferior vena cava filter was placed.

A TEE evaluation of the anesthetized patient was performed pre- and postcardiopulmonary bypass (CPB) using a Philips 7500 ultrasound system (Phillips Medical systems Inc., Andover, MA, USA) equipped with a 4-7 MHz multiplane probe. Consent for intraoperative TEE was obtained as part of the anesthesia consent. The pre-CPB TEE examination demonstrated signs consistent with PA obstruction and RV dysfunction, including an enlarged right atrium and $\mathrm{RV}$, moderate TR, leftward bowing of the interatrial septum, and paradoxical motion of the interventricular septum. Left ventricular (LV) volume was diminished but function was preserved. The PA was dilated, but the borders of the thrombus in the RPA were 


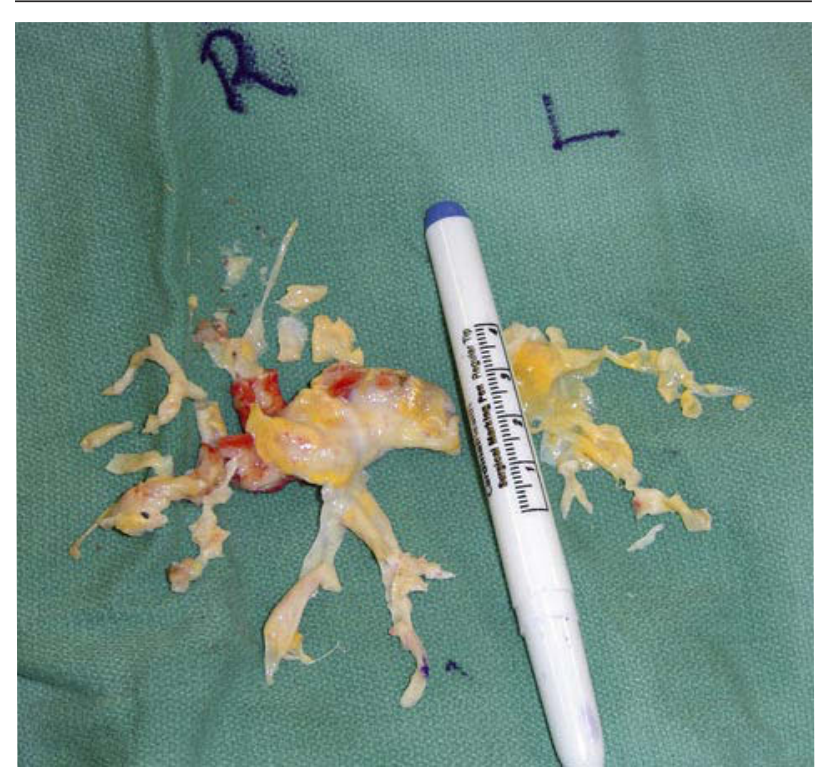

FIGURE 3 Organized thrombus excised from the right pulmonary artery. Minimal tissue was removed from the left pulmonary artery.

only minimally visualized (Figure 1). Perflutren lipid microspheres (Definity ${ }^{\mathrm{TM}}$, Bristol-Myers Squibb, N. Billerica, MA, USA), composed of octafluoropropane encapsulated in an outer lipid shell, were then injected as a $0.3-\mathrm{mL}$ iv bolus into the central venous catheter via the right internal jugular vein while visualizing the RPA with harmonic ultrasound imaging (second harmonic at $5.8 \mathrm{MHz}$ ). The CE-TEE clearly visualized a large mobile thrombus (Figure 2) along with a distinct pattern of swirling flow indicative of PA obstruction. No obstructing embolus was detected in the main PA.

Pulmonary thromboendarterectomy was performed via median sternotomy using $\mathrm{CPB}$. No occlusive thrombus was seen by the surgeon in the left PA but significant tissue was evacuated from RPA (Figure 3). The post-CPB TEE confirmed thrombus evacuation from the RPA, as well as a decrease in the degree of TR. Neither mass nor flow abnormalities were seen on repeat CE-TEE. The patient was successfully weaned from $\mathrm{CPB}$ with a systolic PA pressure of $70 \mathrm{mmHg}$, then transferred to the intensive care unit, and discharged home two weeks later. At five months postsurgery, she could tolerate moderate physical activity without supplemental oxygen. Repeat transthoracic echocardiography at that time showed a moderately enlarged RV with normal function and trivial TR.

\section{Discussion}

This is the first clinical report describing the use of contrast enhanced ultrasonography (CEU) for the intraoperative detection of PE. A few studies have used agitated saline to detect pulmonary flow disturbances associated with an intraluminal mass. ${ }^{8}$ However, saline contrast, because of the tremendous variation in bubble size and stability, does not clearly delineate PA luminal or mass borders, and thus its applicability is limited. On the other hand, the use of CEU leads to a longer lasting and homogeneous distribution of microbubbles, ${ }^{9}$ that enhances border demarcation by creating a "negative contrast" effect, where the area within an artery or chamber is brighter than the darker areas of tissue, plaque, or thrombus. Analogous utility of CEU to improve echocardiographic delineation of LV thrombi was shown in a recent study, ${ }^{10}$ wherein CEU visualized the LV thrombus in $79 \%$ of patients with a primary nondiagnostic bedside transthoracic echocardiography. Contrast enhanced ultrasonography may also facilitate the diagnosis of interatrial and interventricular shunts producing paradoxical embolism. In addition, secondary signs of PE, such as the swirling flow proximal to an obstruction may be seen, as the fast moving homogeneously opacified flow from the RV encounters a portion of relatively sluggish blood flow proximal to the embolus.

The quality of CEU depends upon harmonic imaging which takes advantage of the fact that microbubbles undergo resonant oscillation when exposed to ultrasound and can be induced to nonlinear motion. A consequence of such nonlinear motion is that sound emitted by a bubble in the ultrasound field contains harmonics. By setting the ultrasound system to receive harmonic frequencies (usually at double the transmitted frequency), reflections from the microbubbles can be enhanced selectively over those from surrounding tissues. ${ }^{11}$ In addition, general image quality is enhanced with harmonic imaging as a result of an improvement in lateral resolution and a reduction of side lobe artifact, clutter, and near field haze. ${ }^{11}$ An example of such image enhancement was seen in our patient where the reverberation artifact disappeared with the administration of contrast (Figures 1, 2).

It should be noted that PE is often difficult to diagnose clinically, and no single test is sufficient to identify this condition in all patients. Relying solely upon classical intraoperative monitors for diagnosis is not optimal, since the clinical manifestations of PE are broad, and many of these parameters are obscured in the anesthetized patient. ${ }^{12}$ While imaging modalities remain the mainstay of PE-diagnosis, its applicability to operative settings may be limited. Conventional 
pulmonary digital subtraction angiography was long considered the diagnostic "gold standard", with a sensitivity and specificity above $95 \% .{ }^{13}$ Pulmonary angiography is rarely used now because of its invasiveness, higher cost, and associated incidence of major complication rates and death of $1 \%$ and $0.5 \%$, respectively. ${ }^{13}$ Subsequently, scintillation ventilation-perfusion scanning became the imaging method of choice in patients with suspected PE. Although ventilation-perfusion scans showing normal and high-probability results have significant predictive value, these findings occur in only a minority of patients. ${ }^{14}$ The technique is also time-consuming, and imaging in critically ill patients is difficult. In recent years, spiral CT has gained popularity. Spiral CT has a sensitivity and specificity of about 95\% in detecting PE in the main, lobar, segmental, and subsegmental arteries, and can be performed within $30 \mathrm{sec}^{15}$ Concurrent pelvic and leg imaging to rule out deep venous thrombosis is an added benefit. ${ }^{16}$ Magnetic resonance imaging is another modality, which does not use iodine contrast and does not involve ionizing radiation exposure. For PE detection, magnetic resonance imaging achieves a high sensitivity and specificity of $83 \%$ and $97 \%$ respectively. ${ }^{17}$ The scan can be completed within $30 \mathrm{sec}$, assesses both perfusion and ventilation, and can provide functional assessment of the cardiac chambers and major blood vessels. ${ }^{18}$ Magnetic resonance venography may also be used to evaluate central venous pathology and deep venous thrombosis of the extremities. Limitations on spatial resolution, however, make evaluation of segmental and subsegmental PE difficult. ${ }^{19}$

In cases of massive PE, the sensitivity and specificity of TEE without contrast agents were reported to be as high as $80-97 \%$ and $84-100 \%$, respectively, and virtually comparable with the diagnostic accuracy of CT scanning. ${ }^{4}$ Intraoperatively, however, the sensitivity of TEE to detect PE in patients undergoing emergent pulmonary embolectomy has been reported to be only $46 \%$, while the sensitivity for direct visualization of thromboemboli at any specific location was even lower at $26 \% .^{7}$ The authors attributed this low sensitivity to the difficulty in achieving optimal conditions for echocardiographic interrogation in hemodynamically unstable patients. Important limitations in the diagnostic ability of TEE include the fact that it can visualize only proximal emboli in the main and lobar PA. Furthermore, left PA visualization by TEE is often limited due to interposition of the left mainstem bronchus. However, despite topographical limitations, TEE may be well suited to detect most cases of massive PE. Indeed, in severe PE, the distribution of emboli is most commonly bilateral. ${ }^{8}$
Moreover, since the RPA is in greater flow continuity with the pulmonary trunk, ${ }^{20}$ emboli are consistently found on the right. Also, in massive PE, secondary signs facilitate diagnosis. These include RV dilatation (end-diastolic diameter $>30 \mathrm{~mm}$ ), $\mathrm{RV} / \mathrm{LV}$ diameter $>\mathrm{l}, \mathrm{RV}$ hypokinesis, TR, paradoxical motion of the interventricular septum, and leftward bowing of the interatrial septum., ${ }^{4,21}$ Importantly, the absence of $\mathrm{RV}$ dysfunction effectively eliminates massive $\mathrm{PE}$ as a cause of cardiovascular instability, and requires pursuit of an alternative diagnosis. ${ }^{4}$ Epicardial imaging has also been suggested as an additional tool for PE-visualization, ${ }^{22}$ but is clearly applicable only to open chest procedures. Therefore, imaging techniques such as CE-TEE that improve the diagnostic capabilities of intraoperative TEE are invaluable.

Theoretically, the $i v$ infusion of microbubbles may compromise an already impaired vascular bed via microembolism. However, in an animal model, iv injection of perflutren lipid microspheres did not produce microvascular obstruction, largely because the microvascular rheology of these bubbles is similar to that of red blood cells. ${ }^{23}$ In general, tissue retention and adherence to injured endothelium seems to be more characteristic of albumin-coated microbubbles than lipid-encapsulated microspheres. ${ }^{24}$ An excellent safety profile has been generated for Definity ${ }^{\mathrm{TM}}$ in studies of patients with compromised vascular beds including coronary stenosis, ${ }^{25}$ carotid stenosis ${ }^{26}$ and renal vascular disease. ${ }^{27}$ Similarly, in our patient, we did not observe any untoward effects following the injection of the contrast agent.

In summary, TEE as a rapid, practical, bedside test remains the primary imaging modality for PE diagnosis in the operating room. Transesophageal echocardiography is highly specific, but because of its low sensitivity in operative settings, its utility remains limited. Intraoperative contrast enhanced TEE may decrease operator dependency and increase the resolution necessary to detect central, surgically accessible PE.

\section{References}

1 Dalen JE, Alpert JS. Natural history of pulmonary embolism. Prog Cardiovasc Dis 1975; 17: 259-70.

2 Geerts WH, Pineo GF, Heit JA, et al. Prevention of venous thromboembolism: the Seventh ACCP Conference on Antithrombotic and Thrombolytic Therapy. Chest 2004; 126(3 Suppl): 338S-400.

3 Kuroiwa M, Furuya $H$, Seo N, et al. Incidence and characteristics of perioperative pulmonary thromboembolism in Japan (Japanese). Masui 2004; 53: 454-63.

4 Wood KE. Major pulmonary embolism. Review of a pathophysiologic approach to the golden hour of 
hemodynamically significant pulmonary embolism. Chest 2002; 121: 877-905.

5 Thistlethwaite PA, Madani M, Jamieson SW. Pulmonary thromboendarterectomy surgery. Cardiol Clin 2004; 22: 467-78.

6 Doyle RL, McCrory D, Channick RN, Simonneau G, Conte J. Surgical treatments/interventions for pulmonary arterial hypertension: ACCP evidence-based clinical practice guidelines. Chest 2004; 126(1 Suppl): 63S-71.

7 Rosenberger P, Shernan SK, Body SC, Eltzschig HK. Utility of intraoperative transesophageal echocardiography for diagnosis of pulmonary embolism. Anesth Analg 2004; 99: 12-6.

8 Pruszczyk P, Torbicki A, Pacho R, et al. Noninvasive diagnosis of suspected severe pulmonary embolism: transesophageal echocardiography vs spiral CT. Chest 1997; 112: 722-8.

9 Miller AP, Nanda NC. Contrast echocardiography: new agents. Ultrasound Med Biol 2004; 30: 425-34.

10 Thanigaraj S, Schechtman KB, Perez JE. Improved echocardiographic delineation of left ventricular thrombus with the use of intravenous second-generation contrast image enhancement. J Am Soc Echocardiogr 1999; 12: 1022-6.

11 Ward RP, Mor-Avi V, Lang RM. Assessment of left ventricular function with contrast echocardiography. Cardiol Clin 2004; 22: 211-9.

12 Capan LM, Miller SM. Monitoring for suspected pulmonary embolism. Anesthesiol Clin North America 2001; 19: 673-703.

13 Stein PD, Athanasoulis C, Alavi A, et al. Complications and validity of pulmonary angiography in acute pulmonary embolism. Circulation 1992; 85: 462-8.

14 Anonymous. Value of the ventilation/perfusion scan in acute pulmonary embolism. Results of the prospective investigation of pulmonary embolism diagnosis (PIOPED). The PIOPED investigators. JAMA 1990; 263: 2753-9.

15 Eyer BA, Goodman LR, Washington L. Clinicians' response to radiologists' reports of isolated subsegmental pulmonary embolism or inconclusive interpretation of pulmonary embolism using MDCT. Am J Roentgenol 2005; 184: 623-8.

16 Loud PA, Katz DS, Bruce DA, Klippenstein DL, Grossman $Z D$. Deep venous thrombosis with suspected pulmonary embolism: detection with combined CT venography and pulmonary angiography. Radiology 2001; 219: 498-502.

17 Obno $\Upsilon$, Higashino T, Takenaka D, et al. MR angiography with sensitivity encoding (SENSE) for suspected pulmonary embolism: comparison with MDCT and ventilation-perfusion scintigraphy. Am J Roentgenol
2004; 183: 91-8.

18 Kreitner KF, Ley S, Kauczor HU, et al. Chronic thromboembolic pulmonary hypertension: pre- and postoperative assessment with breath-hold MR imaging techniques. Radiology 2004; 232: 535-43.

19 Kanne JP, Lalani TA. Role of computed tomography and magnetic resonance imaging for deep venous thrombosis and pulmonary embolism. Circulation 2004; 109(Suppl I): I-15-I-21.

20 Russo A, De Luca M, Vigna C, et al. Central pulmonary artery lesions in chronic obstructive pulmonary disease. A transesophageal echocardiography study. Circulation 1999; 100: 1808-15.

21 Vieillard-Baron A, Qanadli SD, Antakly $\Upsilon$, et al. Transesophageal echocardiography for the diagnosis of pulmonary embolism with acute cor pulmonale: a comparison with radiological procedures. Intensive Care Med 1998; 24: 429-33.

22 Tsubo T, Ebina M, Otomo N, Ishibara H, Matsuki A. Accurate detection of pulmonary embolism using epicardial echocardiography during right nephrectomy in a patient with renal cell carcinoma. J Cardiothorac Vasc Anesth 1998; 12: 684-5.

23 Lindner JR, Song J, Jayaweera AR, Sklenar J, Kaul S. Microvascular rheology of definity microbubbles after intra-arterial and intravenous administration. J Am Soc Echocardiogr 2002; 15: 396-403.

24 Tsutsui JM, Xie F, Cano M, et al. Detection of retained microbubbles in carotid arteries with real-time low mechanical index imaging in the setting of endothelial dysfunction. J Am Coll Cardiol 2004; 44: 1036-46.

25 Tsutsui JM, Elhendy A, Xie F, O’Leary EL, McGrain AC, Porter TR. Safety of dobutamine stress real-time myocardial contrast echocardiography. J Am Coll Cardiol 2005; 45: 1235-42.

26 Ohm C, Bendick PJ, Monash J, et al. Diagnosis of total internal carotid occlusions with duplex ultrasound and ultrasound contrast. Vasc Endovascular Surg 2005; 39: 237-43.

27 Blebea J, Zickler R, Volteas N, et al. Duplex imaging of the renal arteries with contrast enhancement. Vasc Endovascular Surg 2003; 37: 429-36. 